\title{
Number of claims and ruin time for a refracted risk process
}

\author{
Yanhong Li* Zbigniew Palmowski ${ }^{\dagger}$ Chunming Zhao ${ }^{\ddagger}$ Chunsheng Zhang ${ }^{\S}$
}

October 21, 2019

\begin{abstract}
In this paper, we consider a classical risk model refracted at given level. We give an explicit expression for the joint density of the ruin time and the cumulative number of claims counted up to ruin time. The proof is based on solving some integro-differential equations and employing the Lagrange's Expansion Theorem.
\end{abstract}

Keywords: threshold dividend strategy, ruin time, number of claims, refracted risk process.

\section{Introduction}

The joint density of the ruin time and the numbers of claims counted until ruin time has been already studied for a classical risk process over last years. Dickson [3] derived special expression for it using probabilistic arguments. Landriault et al. [11] analyzed this object for the Sparre Andersen risk model with the exponential claims. Later Frostig et al. [6] generalized it to the case of a renewal risk model with the phase-type claims and inter-arrival times. The main tool used there was the duality between the risk model and a workload of a single server queueing model. Zhao and Zhang 20] considered a delayed renewal risk model, where the claim size is $\operatorname{Erlang}(n)$ distributed and the interarrival time is assumed to be infinitely divisible.

Our goal is to derive expression for the joint density of the ruin time and the numbers of claims counted until ruin time for a refracted classical risk process (see Kyrianou and Loeffen [9] for a formal definition). It is also called a compound Poisson risk model under a threshold strategy. The latter process is a classical risk process whose dynamic is changed by subtracting off a fixed linear drift whenever the cumulative risk process is above a pre-specified level $b$. This subtracting of the linear drift corresponds to the dividend payments and the considered strategy is also known as a threshold strategy. Dividend strategies for insurance risk models were first proposed by De Finetti $[2]$ to reflect

\footnotetext{
${ }^{*}$ Sichuan University, Chengdu, 610065, China. Email: yanhonglink@qq.com

${ }^{\dagger}$ Faculty of Pure and Applied Mathematics, Wrocław University of Science and Technology, Wyb. Wyspiańskiego 27, 50-370 Wrocław, Poland, e-mail: zbigniew.palmowski@gmail.com

${ }^{\ddagger}$ Department of Statistics, School of Mathematics, Southwest Jiaotong University, Chengdu, Sichuan, 611756, China. Email:cmzhao@swjtu.cn

${ }^{\S}$ School of mathematical Sciences and LPMC Nankai University, Tianjin 300071, China. Email: zhangcs@nankai.edu.cn
} 
more realistically the surplus cash flows in an insurance portfolio. More recently, many kind of risk related quantities under threshold dividend strategies have been studied by Lin and Pavlova [17], Zhu and Yang [22], Lu and Li [14], [15], [16], Badescu, Drekic and Landriault [1], Gao and Yin [7] (see references therein). The case when the drift of the refracted process is disappearing (everything above threshold $b$ is paid as dividends) is called barrier strategy, see Lin et al. [18], Li and Garrido [12], Zhou [21] and in the references therein.

The paper is organized as follows. In Section 2 we define the model we deal with in this paper. In Section 3 we recall properties of the translation operator and the root of the Lundberg fundamental equation. In particular, we introduce the Lagrange's expansion theorem and some notation. In Section 4 we construct two integro-differential equations identifying the joint Laplace transform of joint density of the numbers of claims counted up to ruin time and the ruin time. Analytical solutions of these two integro-differential equations are given in Section 5. Applying the Lagrange's expansion theorem in Section 6 we give the expression for above mentioned density.

\section{Model}

The classical risk process is given by

$$
U(t)=u+c_{1} t-S(t)
$$

where $U(0)=u$ denotes initial capital, $c$ is the premium rate and $S(t)=\sum_{i=1}^{N_{t}} X_{i}$ represents the total amount of claims appeared up to time $t \geq 0$. That is, $\left\{X_{i}\right\}_{\{i \in \mathbb{N}\}}$ are non-negative i.i.d. random variables with pdf $f(x)$ and cdf $F(x)$ and $\left\{N_{t}\right\}_{\{t \geq 0\}}$ is an independent Poisson process with a parameter $\lambda$. To take into account dividend payments paid when regulated process (after deduction of dividends) is above fixed threshold level $b>0$, we consider so-called refracted process given formally for $c_{2}<c_{1}$ by:

$$
d U_{b}(t)= \begin{cases}c_{1} d t-d S(t), & 0 \leq U_{b}(t) \leq b \\ c_{2} d t-d S(t), & U_{b}(t)>b\end{cases}
$$

and $U_{b}(0)=u$. In this case $c_{1}-c_{2}$ denotes intensity of dividend payments, see Figure 1 . 


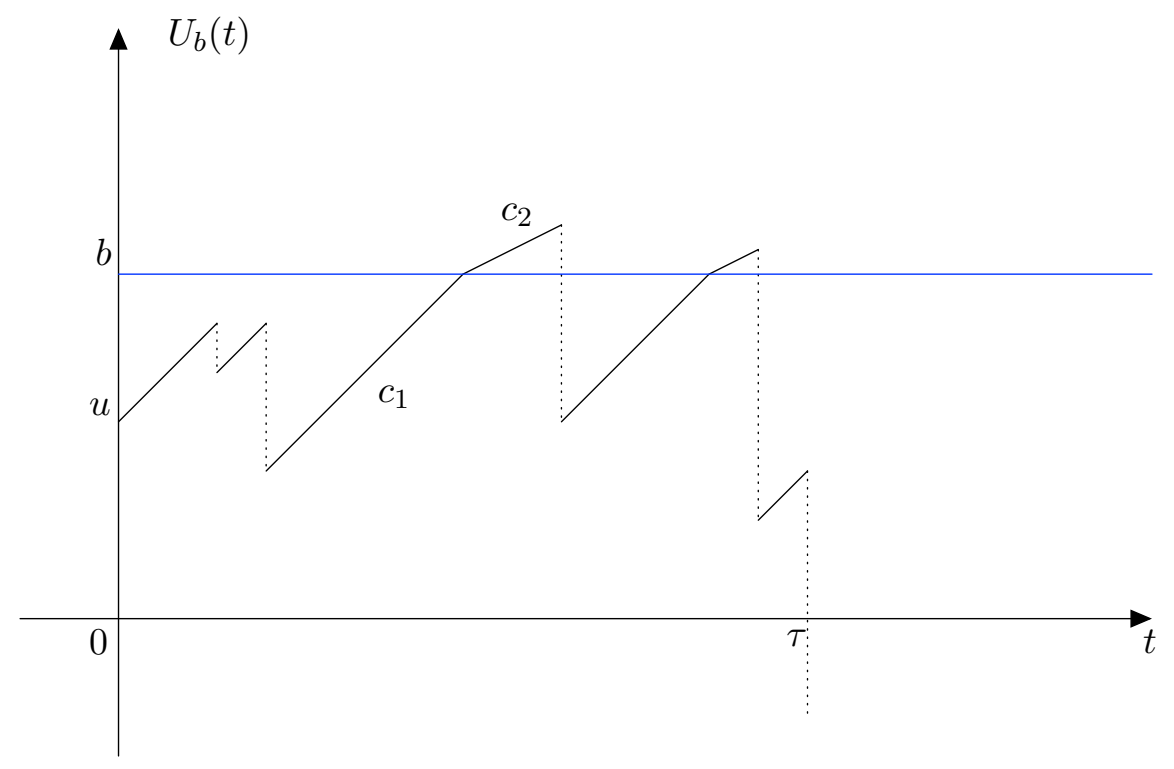

Figure 1: Graphical representation of the surplus process $U_{b}(t)$.

Throughout this paper, we will assume that $c_{2}>\lambda E X_{1}$, which means refracted process $U_{b}(t)$ tends to infinity almost surely. We can then consider the ruin time:

$$
\tau=\inf \left\{t>0, U_{b}(t)<0\right\}
$$

( $\tau=\infty$ if ruin does not occur). Note that $N_{\tau}$ represents the number of claims counted until the ruin time. The main goal of this paper is identification of the density of $\left(\tau, N_{\tau}\right)$. We start from analyzing its Laplace transform:

$$
\begin{aligned}
\phi(u) & =E\left[r^{N_{\tau}} e^{-\delta \tau} \mathbb{I}(\tau<\infty) \mid U_{b}(0)=u\right] \\
& =\sum_{n=1}^{\infty} r^{n} \int_{0}^{\infty} e^{-\delta t} w(u, n, t) d t
\end{aligned}
$$

where

$$
w(u, n, t)=P\left(N_{\tau}=n, \tau \in d t \mid U_{b}(0)=u\right) / d t
$$

is the joint density of $\left(\tau, N_{\tau}\right)$ when $U_{b}(0)=u$. In above definition we have $\delta>0$ and $r \in(0,1]$. Later we will use the following notation

$$
w_{1}(u, n, t)=w(u, n, t) \quad \text { for } u \leq b
$$

and

$$
w_{2}(u, n, t)=w(u, n, t) \quad \text { for } u>b \text {. }
$$




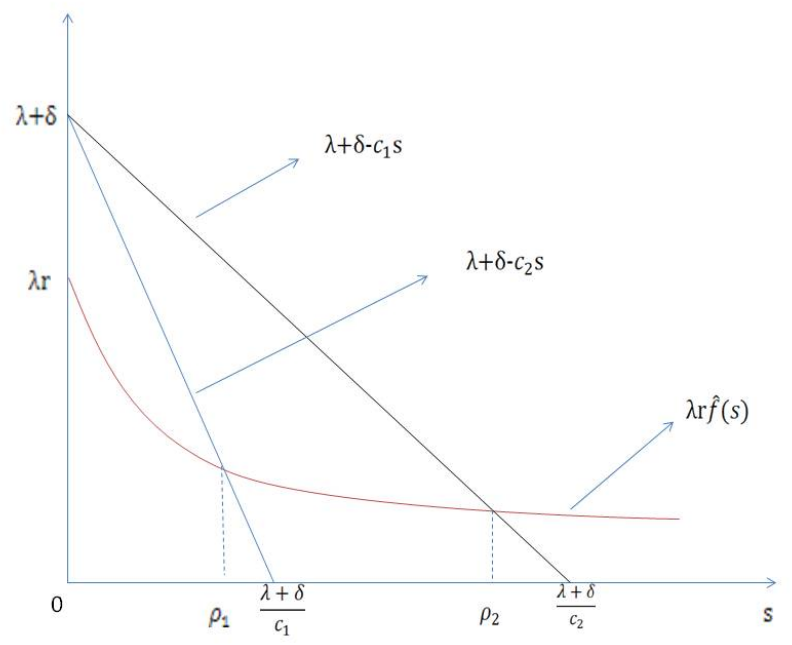

Figure 2: Roots for Lundberg's fundamental equation.

\section{Preliminaries}

In this section we introduce few facts used further in this paper. We start from recalling the translation operator $T_{s}$; see Dickson and Hipp [4]. For any integrable real-valued function $f$ it is defined as

$$
T_{s} f(x)=\int_{x}^{\infty} e^{-s(y-x)} f(y) d y, \quad x \geq 0 .
$$

The operator $T_{s}$ satisfies the following properties:

1. $T_{s} f(0)=\int_{0}^{\infty} e^{-s x} f(x) d x=\hat{f}(s)$ which is the Laplcae transform of $f$;

2. The operator $T_{s}$ is commutative, i.e. $T_{s} T_{r}=T_{r} T_{s}$. Moreover, for $s \neq r$ and $x \geq 0$

$$
T_{s} T_{r} f(x)=T_{r} T_{s} f(x)=\frac{T_{s} f(x)-T_{r} f(x)}{r-s} .
$$

More properties of the translation operator $T_{s}$ can be found in Li and Garrido 13 and Gerber and Shiu 8 .

For any function $g$ we will denote by $\hat{g}(s)$ its Laplace Transform, that is $\hat{g}(s)=\int_{0}^{\infty} e^{-s x} g(x) d x$. Next, for $i=1,2$ let $\rho_{i}$ be the positive root of the Lundberg fundamental equation

$$
c_{i} s-(\lambda+\delta)+\lambda r \hat{f}(s)=0 .
$$

The positive roots always exists for $\delta>0$; see Figure 2 .

Lagrange's Expansion Theorem. In this paper we will also use the Lagrange's Expansion Theorem; see pages 251-326 of Lagrange [10. Given two functions $\alpha(z)$ and $\beta(z)$ which are both analytic on and inside a contour $D$ surrounding a point $a$, if $r$ satisfies the inequality

$$
|r \beta(z)|<|z-a|
$$


for every $z$ on the perimeter of $D$, then $z-a-r \varphi(z)$, as a function of $z$, has exactly one zero $\eta$ in the interior of $D$, and we further have:

$$
\alpha(\eta)=\alpha(a)+\left.\sum_{k=1}^{\infty} \frac{r^{k}}{k !} \frac{d^{k-1}}{d x^{k-1}}\left(\alpha^{\prime}(x) \beta^{k}(x)\right)\right|_{x=a} .
$$

Finally, we define also the impulse function

$$
\delta_{x}(t)= \begin{cases}0, & t \neq x \\ \infty, & t=x\end{cases}
$$

with $\int_{0}^{\infty} \delta_{x}(t) d t=1$. We denote $g^{k *}, k \geq 0$, with $g^{1 *}=g$ and $g^{0 *}(t)=\delta_{0}(t)$ the $k$-fold convolution of $g$ with itself, where

$$
(g * h)(t)=\int_{0}^{t} g(x) h(t-x) d x, \quad t \geq 0
$$

for two functions $g$ and $h$ supported on $(0, \infty)$.

\section{Integro-differential equations for the joint Laplace transform}

In this section, we derive two integro-differential equations identifying $\phi(u)$ defined in (3). We will follow the idea given in Lin and Pavlova [17]. Denote

$$
\phi(u)= \begin{cases}\phi_{1}(u), & u \leq b \\ \phi_{2}(u), & u>b\end{cases}
$$

Theorem 4.1 The joint Laplace transform $\phi$ satisfies the following integro-differential equations:

$$
\left\{\begin{array}{lr}
\phi_{1}^{\prime}(u)=\frac{\lambda+\delta}{c_{1}} \phi_{1}(u)-\frac{\lambda r}{c_{1}} \int_{0}^{u} \phi_{1}(u-x) f(x) d x-\frac{\lambda r}{c_{1}} \bar{F}(u), & 0 \leq u \leq b \\
\phi_{2}^{\prime}(u)=\frac{\lambda+\delta}{c_{2}} \phi_{2}(u)-\frac{\lambda r}{c_{2}}\left(\int_{0}^{u-b} \phi_{2}(u-x) f(x) d x+\int_{u-b}^{u} \phi_{1}(u-x) f(x) d x\right)-\frac{\lambda r}{c_{2}} \bar{F}(u), u>b
\end{array}\right.
$$

with the boundary condition

$$
\phi_{1}(b)=\phi_{2}(b):=\lim _{u \rightarrow b^{+}} \phi_{2}(u) .
$$

Remark 4.2 Note that from the integro-differential equations 12 follows that the joint Laplace transform with initial surplus above the barrier depends on the respective function with initial surplus below the barrier, but the reverse relationship does not hold true.

Proof. Let first $0 \leq u \leq b$. Then conditioning on the occurrence of the first claim we will have two cases: the first claim occurs before the surplus has reached the barrier level $b$ or it occurs after reaching this barrier. There are also two other cases at the moment of the arrival of the first claim: either the risk process starts all over again with new initial surplus or the first claim leads already to ruin. Hence:

$\phi(u)=\phi_{1}(u)$ 


$$
\begin{aligned}
= & \int_{0}^{\frac{b-u}{c_{1}}} \lambda r e^{-\lambda t} e^{-\delta t}\left(\int_{0}^{u+c_{1} t} \phi\left(u+c_{1} t-x\right) f(x) d x+\bar{F}\left(u+c_{1} t\right)\right) d t \\
& +\int_{\frac{b-u}{c_{1}}}^{\infty} \lambda r e^{-\lambda t} e^{-\delta t}\left(\int_{0}^{b+c_{2}\left(t-\frac{b-u}{c_{1}}\right)} \phi\left(b+c_{2}\left(t-\frac{b-u}{c_{1}}\right)-x\right) f(x) d x+\bar{F}\left(b+c_{2}\left(t-\frac{b-u}{c_{1}}\right)\right)\right) d t \\
= & \lambda r \int_{0}^{\frac{b-u}{c_{1}}} e^{-(\lambda+\delta) t} \gamma\left(u+c_{1} t\right) d t+\lambda r \int_{\frac{b-u}{c_{1}}}^{\infty} e^{-(\lambda+\delta) t} \gamma\left(b+c_{2}\left(t-\frac{b-u}{c_{1}}\right) d t,\right.
\end{aligned}
$$

where $\gamma(t)=\int_{0}^{t} \phi(t-x) f(x) d x+\bar{F}(t)$.

Changing variables in (14) and rearranging leads to the following equation for $0 \leq u \leq b$ :

$$
\phi_{1}(u)=\frac{\lambda r}{c_{1}} e^{(\lambda+\delta) u / c_{1}} \int_{u}^{b} e^{-(\lambda+\delta) t / c_{1}} \gamma(t) d t+\frac{\lambda r}{c_{2}} e^{(\lambda+\delta) u / c_{1}} \int_{b}^{\infty} e^{-(\lambda+\delta)\left[t-\left(c_{1}-c_{2}\right) b / c_{1}\right] / c_{2}} \gamma(t) d t .
$$

Differentiating both sides of (15) with respect to $u$ yields first equation.

Similarly, for $u>b$ we have:

$$
\begin{aligned}
\phi(u) & =\phi_{2}(u) \\
& =\int_{0}^{\infty} \lambda r e^{-\lambda t} e^{-\delta t}\left(\int_{0}^{u+c_{2} t} \phi\left(u+c_{2} t-x\right) f(x) d x+\bar{F}\left(u+c_{2} t\right)\right) d t \\
& =\lambda r \int_{0}^{\infty} e^{-(\lambda+\delta) t} \gamma\left(u+c_{2} t\right) d t \\
& =\frac{\lambda r}{c_{2}} e^{(\lambda+\delta) u / c_{2}} \int_{u}^{\infty} e^{-(\lambda+\delta) t / c_{2}} \gamma(t) d t .
\end{aligned}
$$

Differentiating both sides of (16) with respect to $u$ produces the second equation.

Note also that from equations (15) and (16) it follows that $\phi(u)$ is continuous at $u=b$ and hence (13) holds. This completes the proof.

\section{The analytical expression for $\phi(u)$}

In this section, we derive the analytical expression for $\phi_{i}(u)(i=1,2)$ using the translation operator introduced in Section 3.

Theorem 5.1 The function $\phi_{2}(u)$ can be expressed analytically as follows:

$$
\phi_{2}(u)=\sum_{n=0}^{\infty}\left(\frac{\lambda r}{c_{2}}\right)^{n+1}\left(T_{\rho_{2}} f\right)^{n *} * h(u-b), \quad u>b,
$$

where

$$
h(u):=\int_{u}^{u+b} \phi_{1}(u+b-x) T_{\rho_{2}} f(x) d x+T_{\rho_{2}} \bar{F}(u+b) .
$$

Proof. We adopt the approach of Willmot and Dickson [19]. Consider the second equation in (12) for $u>b$. For a fixed $s>0$, we multiply both sides of this equation by $e^{-s(u-b)}$ and integrate it with respect to $u$ from $b$ to $\infty$ :

$$
c_{2} \int_{b}^{\infty} e^{-s(u-b)} \phi_{2}^{\prime}(u) d u=(\lambda+\delta) T_{s} \phi_{2}(b)-\lambda r \int_{b}^{\infty} e^{-s(u-b)} \int_{0}^{u-b} \phi_{2}(u-x) f(x) d x d u
$$




$$
\begin{aligned}
& -\lambda r \int_{b}^{\infty} e^{-s(u-b)} \int_{0}^{b} \phi_{1}(y) f(u-y) d y d u-\lambda r T_{s} \bar{F}(b) \\
= & (\lambda+\delta) T_{s} \phi_{2}(b)-\lambda r \int_{0}^{\infty} e^{-s x} f(x) \int_{x+b}^{\infty} e^{-s(u-x-b)} \phi_{2}(u-x) d u d x \\
& -\lambda r \int_{0}^{b} \phi_{1}(y) \int_{b}^{\infty} e^{-s(u-b)} f(u-y) d u d y-\lambda r T_{s} \bar{F}(b) \\
= & (\lambda+\delta) T_{s} \phi_{2}(b)-\lambda r \hat{f}(s) T_{s} \phi_{2}(b)-\lambda r \int_{0}^{b} \phi_{1}(y) T_{s} f(b-y) d y-\lambda r T_{s} \bar{F}(b) .
\end{aligned}
$$

Integrating by parts gives:

$$
c_{2} \int_{b}^{\infty} e^{-s(u-b)} \phi_{2}^{\prime}(u) d u=c_{2} s T_{s} \phi_{2}(b)-c_{2} \phi_{2}(b) .
$$

Hence

$$
c_{2} s T_{s} \phi_{2}(b)-c_{2} \phi_{2}(b)=(\lambda+\delta) T_{s} \phi_{2}(b)-\lambda r \hat{f}(s) T_{s} \phi_{2}(b)-\lambda r \int_{0}^{b} \phi_{1}(y) T_{s} f(b-y) d y-\lambda r T_{s} \bar{F}(b)
$$

and simple rearranging leads to:

$$
\left(c_{2} s-(\lambda+\delta)+\lambda r \hat{f}(s)\right) T_{s} \phi_{2}(b)=c_{2} \phi_{2}(b)-\lambda r \int_{0}^{b} \phi_{1}(y) T_{s} f(b-y) d y-\lambda r T_{s} \bar{F}(b) .
$$

Taking $s=\rho_{2}$ for the solution $\rho_{2}$ of the Lundberg Fundamental Equation (8) gives

$$
c_{2} \phi_{2}(b)=\lambda r \int_{0}^{b} \phi_{1}(y) T_{\rho_{2}} f(b-y) d y+\lambda r T_{\rho_{2}} \bar{F}(b) .
$$

Then equation 19 is equivalent to:

$$
\left[c_{2}\left(s-\rho_{2}\right)+\lambda r \hat{f}(s)-\lambda r \hat{f}\left(\rho_{2}\right)\right] T_{s} \phi_{2}(b)=\lambda r \int_{0}^{b} \phi_{1}(y)\left[T_{\rho_{2}} f(b-y)-T_{s} f(b-y)\right] d y+\lambda r\left[T_{\rho_{2}} \bar{F}(b)-T_{s} \bar{F}(b)\right] .
$$

Now dividing above equation by $s-\rho_{2}$ and using property 2 of the translation operator introduced in Section 2 produces:

$$
c_{2} T_{s} \phi_{2}(b)=\lambda r T_{s} T_{\rho_{2}} f(0) T_{s} \phi_{2}(b)+\lambda r \int_{0}^{b} \phi_{1}(y) T_{s} T_{\rho_{2}} f(b-y) d y+\lambda r T_{s} T_{\rho_{2}} \bar{F}(b) .
$$

Inverting the translation operators of 20 yields the following renewal equation for $\phi_{2}(u)$ :

$$
\phi_{2}(u)=\frac{\lambda r}{c_{2}}\left[\int_{0}^{u-b} \phi_{2}(u-x) T_{\rho_{2}} f(x) d x+\int_{u-b}^{u} \phi_{1}(u-x) T_{\rho_{2}} f(x) d x+T_{\rho_{2}} \bar{F}(u)\right] .
$$

Taking $y=u-b$ and $g(y)=\phi_{2}(y+b)$ we can rewrite 21) as follows:

$$
g(y)=\frac{\lambda r}{c_{2}} \int_{0}^{y} g(y-x) T_{\rho_{2}} f(x) d x+\frac{\lambda r}{c_{2}} h(y), \quad y>0
$$

where

$$
h(y)=h(u-b)=\int_{u-b}^{u} \phi_{1}(u-x) T_{\rho_{2}} f(x) d x+T_{\rho_{2}} \bar{F}(u), \quad u>b .
$$


Hence

$$
\begin{aligned}
\phi_{2}(u) & =g(y) \\
& =\frac{\lambda r}{c_{2}} \int_{0}^{y} g(y-x) T_{\rho_{2}} f(x) d x+\frac{\lambda r}{c_{2}} h(y) \\
& =\sum_{n=0}^{\infty}\left(\frac{\lambda r}{c_{2}}\right)^{n+1}\left(T_{\rho_{2}} f\right)^{n *} * h(y) \\
& =\sum_{n=0}^{\infty}\left(\frac{\lambda r}{c_{2}}\right)^{n+1}\left(T_{\rho_{2}} f\right)^{n *} * h(u-b)
\end{aligned}
$$

which completes the proof.

The expression for $\phi_{1}(u)$ could be also derived in terms of the translation operator.

Theorem 5.2 The function $\phi_{1}(u)$ can be expressed analytically in the following form:

$$
\phi_{1}(u)=\phi_{\infty}(u)+\frac{\frac{\lambda r}{c_{2}}\left[\phi_{\infty} * T_{\rho_{2}} f(b)+T_{\rho_{2}} \bar{F}(b)\right]-\phi_{\infty}(b)}{\nu(b)-\frac{\lambda r}{c_{2}} \nu * T_{\rho_{2}} f(b)} \nu(u),
$$

where

$$
\phi_{\infty}(u):=\sum_{n=0}^{\infty}\left(\frac{\lambda r}{c_{1}}\right)^{n+1}\left(T_{\rho_{1}} f\right)^{n *} * T_{\rho_{1}} \bar{F}(u)
$$

and

$$
\nu(x):=\sum_{n=0}^{\infty}\left(\frac{\lambda r}{c_{1}}\right)^{n}\left(T_{\rho_{1}} f\right)^{n *} * p(x)
$$

with $p(x)=e^{\rho_{1} x}$.

Proof. Note that the first equation in $(12)$ does not involve the barrier level $b$ :

$$
\phi_{1}^{\prime}(u)=\frac{\lambda+\delta}{c_{1}} \phi_{1}(u)-\frac{\lambda r}{c_{1}} \int_{0}^{u} \phi_{1}(u-x) f(x) d x-\frac{\lambda r}{c_{1}} \bar{F}(u) .
$$

The information about the barrier $b$ is included in the boundary condition:

$$
\phi_{1}(b)=\phi_{2}(b):=\lim _{u \rightarrow b^{+}} \phi_{2}(u) .
$$

Lin et al. [17] showed that the general solution of (25) is of the form

$$
\phi_{1}(u)=\phi_{\infty}(u)+k \nu(u)
$$

where $\phi_{\infty}(u)$ is the joint Laplace transform of density of the ruin time and number of claims counted up to ruin time for the classical risk process (1) without any barrier applied. That is,

$$
\phi_{\infty}(u):=\sum_{n=1}^{\infty} r^{n} \int_{0}^{\infty} e^{-\delta t} w_{\infty}(u, n, t) d t
$$

for

$$
w_{\infty}(u, n, t):=P\left(N_{\tau}=n, \tau \in d t \mid U(0)=u\right) / d t
$$


In above equation (26) the quantity $k$ is a constant which we can specify by implementing (26) and (21):

$$
k=\frac{\frac{\lambda r}{c_{2}}\left[\int_{0}^{b} \phi_{\infty}(b-x) T_{\rho_{2}} f(x) d x+T_{\rho_{2}} \bar{F}(b)\right]-\phi_{\infty}(b)}{\nu(b)-\frac{\lambda r}{c_{2}} \int_{0}^{b} \nu(b-x) T_{\rho_{2}} f(x) d x} .
$$

We express now the function $\phi_{\infty}$ in terms of a compound geometric distribution. Indeed, since $\phi_{\infty}$ also satisfies equation (25), taking Laplace transforms of its both sides for sufficiently large $s$ gives:

$$
\left(c_{1} s-(\lambda+\delta)+\lambda r \hat{f}(s)\right) \hat{\phi}_{\infty}(s)=c_{1} \phi_{\infty}(0)-\lambda r \hat{\bar{F}}(s), s \geq 0 .
$$

To determine the constant term $c_{1} \phi_{\infty}(0)$ in $(30)$, we substitute the solution $\rho_{1}$ of the Lundberg Fundamental Equation (8) for $s$ :

$$
c_{1} \phi_{\infty}(0)=\lambda r \hat{\bar{F}}\left(\rho_{1}\right)=\lambda r T_{\rho_{1}} \hat{\bar{F}}(0) .
$$

Consequently, the equation 30 reduces to

$$
\left[c_{1}\left(s-\rho_{1}\right)+\lambda r \hat{f}(s)-\lambda r \hat{f}\left(\rho_{1}\right)\right] \hat{\phi}_{\infty}(s)=\lambda r \hat{\bar{F}}\left(\rho_{1}\right)-\lambda r \hat{\bar{F}}(s) .
$$

Dividing above equation by $s-\rho_{1}$ and simple rearranging along with implementation of the formula (7) produces:

$$
c_{1} \hat{\phi}_{\infty}(s)=\lambda r \hat{\phi}_{\infty}(s) T_{s} T_{\rho_{1}} f(0)+\lambda r T_{s} T_{\rho_{1}} \bar{F}(0) .
$$

Inverting this Laplace transforms gives classical renewal equation:

$$
\phi_{\infty}(u)=\frac{\lambda r}{c_{1}} \phi_{\infty} * T_{\rho_{1}} f(u)+\frac{\lambda r}{c_{1}} T_{\rho_{1}} \bar{F}(u)
$$

having the solution given as an Neumann infinite series 23.

To prove the last statement (24) note that the function $\nu(u)$ satisfies the following integrodifferential equation:

$$
c_{1} \nu^{\prime}(u)-(\lambda+\delta) \nu(u)+\lambda r \int_{0}^{u} \nu(u-x) f(x) d x=0, \quad u \geq 0,
$$

with the initial condition $\nu(0)=1$. To get the analytical expression of $\nu(u)$ we take the Laplace transforms of both sides of $(33)$ for sufficiently large $s\left(s>\rho_{1}\right)$. This yields:

$$
c_{1} s \hat{\nu}(s)-c_{1} \nu(0)=(\lambda+\delta) \hat{\nu}(s)-\lambda r \hat{f}(s) \hat{\nu}(s) .
$$

Since $\nu(0)=1$,

$$
\left(s+\frac{\lambda r}{c_{1}} \hat{f}(s)-\frac{\lambda+\delta}{c_{1}}\right) \hat{\nu}(s)=1
$$

Recalling that $\rho_{1}$ is the root of (8), we can rewrite (34) as

$$
\left(s-\rho_{1}+\frac{\lambda r}{c_{1}}\left[\hat{f}(s)-\hat{f}\left(\rho_{1}\right)\right]\right) \hat{\nu}(s)=1,
$$

which, by dividing by $s-\rho_{1}$ and implementing (7), produces:

$$
\hat{\nu}(s)=\frac{\lambda r}{c_{1}} \hat{\nu}(s) T_{s} T_{\rho_{1}} f(0)+\frac{1}{s-\rho_{1}} .
$$

Inverting the Laplace transforms in (35) leads to the equation (24). Including all above identities in (26) completes the proof. 


\section{The joint density of $\left(\tau, N_{\tau}\right)$}

In this section we give the joint density of the number of claims counted until ruin time and the ruin time using the Lagrange's Expansion theorem. We start with few facts that will be useful in the proof of the main result.

Recall that by $w_{\infty}(u, n, t)$ we denote the joint density of $\left(\tau, N_{\tau}\right)$ for the classical risk process (1) (with infinite barrier $b=+\infty$ ); see $(28)$. For $i=1,2$ we denote

$$
\begin{aligned}
g_{i}(x, 0, t) & :=\delta_{x / c_{i}}(t) e^{-\lambda x / c_{i}} \\
g_{i}(x, n, t) & :=x t^{n-1} e^{-\lambda t} \lambda^{n} f^{n *}\left(c_{i} t-x\right) / n ! .
\end{aligned}
$$

Lemma 6.1 We have

$$
w_{\infty}(u, 1, t)=\lambda e^{-\lambda t} \bar{F}\left(u+c_{1} t\right) .
$$

For $n=1,2,3, \ldots$ the following holds:

$$
\begin{aligned}
w_{\infty}(u, n+1, t)= & \frac{(\lambda t)^{n}}{n !} e^{-\lambda t} \int_{0}^{u+c_{1} t} f^{n *}\left(u+c_{1} t-x\right) \lambda \bar{F}(x) d x \\
& \left.-c_{1} \sum_{j=1}^{n} \int_{0}^{t} \frac{(\lambda s)^{j}}{j !} e^{-\lambda s} f^{j *}\left(u+c_{1} s\right) w_{\infty}(0, n+1-j, t-s)\right) d s
\end{aligned}
$$

where

$$
w_{\infty}(0, n, t)=\frac{\lambda}{c_{1}} \int_{0}^{c_{1} t} \bar{F}(x) g_{1}(x, n-1, t) d x, \quad n=1,2, \ldots
$$

Proof. Using Lagrange's Expansion Theorem presented in Section 2 with $\alpha(z)=e^{-z x}, \beta(z)=$ $-\frac{\lambda}{c_{i}} \hat{f}(s), a=(\lambda+\delta) / c_{i}$ and $D=\{z|| z-a \mid \leq a\} \quad(i=1,2)$ and the Lundberg fundamental equation (8) we can conclude the following identity:

$$
\begin{aligned}
e^{-\rho_{i} x} & =e^{-(\lambda+\delta) x / c_{i}}+\left.\sum_{n=1}^{\infty} \frac{r^{n}}{n !} \frac{d^{n-1}}{d s^{n-1}}\left(-x e^{-s x}\left(-\frac{\lambda}{c_{i}} \hat{f}(s)\right)^{n}\right)\right|_{s=(\lambda+\delta) / c_{i}} \\
& =e^{-(\lambda+\delta) x / c_{i}}+\left.\sum_{n=1}^{\infty} \frac{r^{n}}{n !} \frac{d^{n-1}}{d s^{n-1}}\left((-1)^{n+1} \lambda^{n} x / c_{i}^{n} \int_{0}^{\infty} e^{-s(x+y)} f^{n *}(y) d y\right)\right|_{s=(\lambda+\delta) / c_{i}} \\
& =e^{-(\lambda+\delta) x / c_{i}}+\sum_{n=1}^{\infty} \frac{\lambda^{n} r^{n}}{n ! c_{i}^{n}} \int_{0}^{\infty} x(x+y)^{n-1} e^{-(\lambda+\delta)(x+y) / c_{i}} f^{n *}(y) d y
\end{aligned}
$$

Substituting $t:=(x+y) / c_{i}$ and rearranging leads to:

$$
\begin{aligned}
e^{-\rho_{i} x} & =e^{-(\lambda+\delta) x / c_{i}}+\sum_{n=1}^{\infty} r^{n} \frac{\lambda^{n}}{n !} \int_{x / c_{i}}^{\infty} x t^{n-1} e^{-\lambda t} e^{-\delta t} f^{n *}\left(c_{i} t-x\right) d t \\
& =\sum_{n=0}^{\infty} r^{n} \int_{x / c_{i}}^{\infty} e^{-\delta t} g_{i}(x, n, t) d t .
\end{aligned}
$$

Therefore,

$$
T_{\rho_{i}} f(x)=\int_{x}^{\infty} e^{-\rho_{i}(u-x)} f(u) d u
$$




$$
\begin{aligned}
& =\int_{x}^{\infty} \sum_{n=0}^{\infty} r^{n} \int_{(u-x) / c_{i}}^{\infty} e^{-\delta t} g_{i}(u-x, n, t) d t f(u) d u \\
& =\sum_{n=0}^{\infty} r^{n} \int_{0}^{\infty} e^{-\delta t} \int_{x}^{c_{i} t+x} f(u) g_{i}(u-x, n, t) d u d t
\end{aligned}
$$

Since $\phi_{\infty}(u)$ defined in 27$)$ is the joint Laplace transform under the classical compound Poisson risk model without a barrier we can use Dickson 3 to complete the proof.

Lemma 6.2 The function $\nu(u)$ given in 24) equals

$$
\nu(u)=\sum_{n=0}^{\infty} r^{n} \int_{0}^{\infty} e^{-\delta t} \varpi(u, n, t) d t,
$$

where

$$
\begin{aligned}
\varpi(u, 0, t) & :=g_{1}(-u, 0, t), \\
\varpi(u, n, t) & :=\sum_{m=1}^{n}\left(\frac{\lambda}{c_{1}}\right)^{m} \int_{0}^{c_{1} t} \int_{0}^{u} g_{c_{1}}(y, n-m, t) b_{m}(u-x, y+x) d x d y+g_{c_{1}}(-u, n, t), \quad n \geq 1 \\
b_{n}(u, y) & :=\sum_{j=0}^{n-1}\left(\begin{array}{c}
n \\
j
\end{array}\right) \frac{(-1)^{j}}{\Gamma(n)} \int_{0}^{u}(u-x)^{n-1} f^{(n-j) *}(y+u-x) f^{j *}(x) d x .
\end{aligned}
$$

Proof. Our goal is to express $\nu(u)$ as the Laplace transform:

$$
\nu(u)=\int_{0}^{\infty} e^{-\rho_{1} t} \xi(u, t) d t .
$$

We start from definition (24):

$$
\begin{aligned}
\nu(u) & =\sum_{n=0}^{\infty}\left(\frac{\lambda r}{c_{1}}\right)^{n}\left(T_{\rho_{1}} f\right)^{n *} * p(u) \\
& =\sum_{n=1}^{\infty}\left(\frac{\lambda r}{c_{1}}\right)^{n} \int_{0}^{u}\left(T_{\rho_{1}} f\right)^{n *}(u-x) e^{\rho_{1} x} d x+e^{\rho_{1} u} .
\end{aligned}
$$

Using Dickson and Willmot [5] we can obtain the following representation:

$$
\left(T_{\rho_{i}} f\right)^{n *}(u)=\int_{0}^{\infty} e^{-\rho_{i} y} b_{n}(u, y) d y
$$

for

$$
b_{n}(u, y):=\sum_{j=0}^{n-1}\left(\begin{array}{l}
n \\
j
\end{array}\right) \frac{(-1)^{j}}{\Gamma(n)} \int_{0}^{u}(u-x)^{n-1} f^{(n-j) *}(y+u-x) f^{j *}(x) d x .
$$

By 42

$$
\begin{aligned}
\nu(u) & =\sum_{n=1}^{\infty}\left(\frac{\lambda r}{c_{1}}\right)^{n} \int_{0}^{u} \int_{0}^{\infty} e^{-\rho_{1} y} b_{n}(u-x, y) d y e^{\rho_{1} x} d x+e^{\rho_{1} u} \\
& =\sum_{n=1}^{\infty}\left(\frac{\lambda r}{c_{1}}\right)^{n} \int_{0}^{\infty} e^{-\rho_{1} t} \int_{0}^{u} b_{n}(u-x, t+x) d x d t
\end{aligned}
$$




$$
+\sum_{n=1}^{\infty}\left(\frac{\lambda r}{c_{1}}\right)^{n} \int_{-u}^{0} e^{-\rho_{1} t} \int_{-t}^{u} b_{n}(u-x, t+x) d x d t+\int_{0}^{\infty} e^{-\rho_{1} t} \delta_{-u}(t) d t .
$$

Comparing the coefficients of $e^{-\rho_{1} t}$ in 41 gives:

$$
\xi(u, t)=\sum_{n=1}^{\infty}\left(\frac{\lambda r}{c_{1}}\right)^{n} \int_{0}^{u} b_{n}(u-x, t+x) d x+\delta_{-u}(t) .
$$

Using (38) and 44 in (41) we end up with:

$$
\begin{aligned}
\nu(u)= & \int_{0}^{\infty} e^{-\rho_{1} y} \xi(u, y) d y+e^{\rho_{1} u} \\
= & \int_{0}^{\infty} \sum_{n=0}^{\infty} r^{n} \int_{y / c_{1}}^{\infty} e^{-\delta t} g_{c_{1}}(y, n, t) d t \xi(u, y) d y \\
= & \sum_{n=0}^{\infty} r^{n} \int_{0}^{\infty} e^{-\delta t} \int_{0}^{c_{1} t} g_{c_{1}}(y, n, t) \xi(u, y) d y d t \\
= & \sum_{n=1}^{\infty} r^{n} \int_{0}^{\infty} e^{-\delta t}\left(\sum_{m=1}^{n}\left(\frac{\lambda}{c_{1}}\right)^{m} \int_{0}^{c_{1} t} \int_{0}^{u} g_{c_{1}}(y, n-m, t) b_{m}(u-x, y+x) d x d y+g_{c_{1}}(-u, n, t)\right) d t \\
& +\int_{0}^{\infty} e^{-\delta t} g_{c_{1}}(-u, 0, t) d t
\end{aligned}
$$

which completes the proof.

Using above lemmas we will prove the main result of this paper.

Theorem 6.3 For $0 \leq u \leq b$ and $m>1$ the joint density of the number of claims until ruin $N_{\tau}$ and the time to ruin $\tau$ is given by

$$
\begin{aligned}
& w_{1}(u, 1, t)=\frac{\lambda}{c_{2}} e^{-\lambda t} \bar{F}\left(c_{2} t+b+\frac{c_{2}}{c_{1}}(u-b)\right) \\
& w_{1}(u, m, t)=e^{-\frac{\lambda b}{c_{1}}}\left[\sum_{n=1}^{m} \vartheta\left(u, m, n, t-\frac{b}{c_{1}}\right)-\sum_{n=1}^{m-1} \int_{0}^{t-\frac{b}{c_{1}}} \varsigma\left(b, m-n, t-\frac{b}{c_{1}}-z\right) w_{1}(u, n, z) d z\right],
\end{aligned}
$$

where for $n \geq 1$

$$
\begin{aligned}
& \varsigma(b, 0, t):=\varpi(b, 0, t)=g_{c_{1}}(-b, 0, t), \\
& \varsigma(b, n, t):=\varpi(b, n, t)-\sum_{m=0}^{n-1} \frac{\lambda}{c_{2}} \int_{0}^{b} \int_{0}^{t} \varpi(b-x, n-1-m, t-z) \int_{x}^{c_{2} z+x} f(y) g_{2}(y-x, m, z) d y d z d x, \\
& \gamma(b, 1, t):=\frac{\lambda}{c_{2}} \int_{b}^{c_{2} t+b} \bar{F}(y) g_{2}(y-b, 0, t) d y, \\
& \gamma(b, n, t):=\sum_{m=0}^{n-2} \frac{\lambda}{c_{2}} \int_{0}^{b} \int_{0}^{t} w_{\infty}(b-x, n-m-1, t-z) \int_{x}^{c_{2} z+x} f(y) g_{2}(y-x, m, z) d y d z d x, \\
& \vartheta(u, m, n, t):=\int_{0}^{t} \varsigma(b, m-n, t-z) w_{\infty}(u, n, z)+\left(\gamma(b, n, t-z)-w_{\infty}(b, n, t-z)\right) \varpi(u, m-n, z) d z .
\end{aligned}
$$


Proof. In order to get the joint density $w(u, n, t)$, we have to take inverse Laplace transform with respect to $\delta$ rather than $\rho_{1}$ and $\rho_{2}$. To do this we must find firstly the relationship between transforms with respect to $\rho_{1}, \rho_{2}$ and $\delta$ by applying the Lagrange's Expansion theorem. For convenience, we will denote:

$$
\chi(b):=\nu(b)-\frac{\lambda r}{c_{2}} \nu * T_{\rho_{2}} f(b) .
$$

Then we can rewrite 22 as follows:

$$
\chi(b) \phi_{1}(u)=\chi(b) \phi_{\infty}(u)+\frac{\lambda r}{c_{2}}\left[\phi_{\infty} * T_{\rho_{2}} f(b)+T_{\rho_{2}} \bar{F}(b)\right] \nu(u)-\phi_{\infty}(b) \nu(u) .
$$

Putting (39) and 40 into 46) we will derive:

$$
\begin{aligned}
\chi(b)= & \sum_{n=0}^{\infty} r^{n} \int_{0}^{\infty} e^{-\delta t} \varpi(b, n, t) d t-\frac{\lambda r}{c_{2}} \int_{0}^{b} \nu(b-x) T_{\rho_{2}} f(x) d x \\
= & \sum_{n=0}^{\infty} r^{n} \int_{0}^{\infty} e^{-\delta t} \varpi(b, n, t) d t-\frac{\lambda r}{c_{2}} \sum_{n=0}^{\infty} r^{n} \sum_{m=0}^{n} \int_{0}^{b} \int_{0}^{\infty} e^{-\delta t} \varpi(b-x, n-m, t) d t \int_{0}^{\infty} e^{-\delta z} \\
& \int_{x}^{c_{2} z+x} f(y) g_{2}(y-x, m, z) d y d z d x \\
= & \sum_{n=0}^{\infty} r^{n} \int_{0}^{\infty} e^{-\delta t} \varpi(b, n, t) d t-\sum_{n=1}^{\infty} r^{n} \int_{0}^{\infty} e^{-\delta t}\left\{\sum_{m=0}^{n-1} \frac{\lambda}{c_{2}} \int_{0}^{b} \int_{0}^{t} \varpi(b-x, n-1-m, t-z)\right. \\
& \left.\int_{x}^{c_{2} z+x} f(y) g_{2}(y-x, m, z) d y d z d x\right\} d t \\
= & \sum_{n=1}^{\infty} r^{n} \int_{0}^{\infty} e^{-\delta t}\left\{\varpi(b, n, t)-\sum_{m=0}^{n-1} \frac{\lambda}{c_{2}} \int_{0}^{b} \int_{0}^{t} \varpi(b-x, n-1-m, t-z) \int_{x}^{c_{2} z+x} f(y)\right. \\
& \left.g_{2}(y-x, m, z) d y d z d x\right\} d t+\int_{0}^{\infty} e^{-\delta t} \varpi(b, 0, t) d t \\
= & \sum_{n=0}^{\infty} r^{n} \int_{0}^{\infty} e^{-\delta t} \varsigma(b, n, t) d t .
\end{aligned}
$$

Similarly, using Lemma 6.1, we can check that:

$$
\frac{\lambda r}{c_{2}}\left[\phi_{\infty} * T_{\rho_{2}} f(b)+T_{\rho_{2}} \bar{F}(b)\right]=\sum_{n=1}^{\infty} r^{n} \int_{0}^{\infty} e^{-\delta t} \gamma(b, n, t) d t .
$$

Using (40), 48) and (49) in (47) we obtain:

$$
\begin{aligned}
& \sum_{m=1}^{\infty} r^{m} \int_{0}^{\infty} e^{-\delta t} \sum_{n=1}^{m} \int_{0}^{t} \varsigma(b, m-n, t-z)\left(w_{1}(u, n, z)-w_{\infty}(u, n, z)\right) d z d t \\
= & \sum_{m=1}^{\infty} r^{m} \int_{0}^{\infty} e^{-\delta t} \sum_{n=1}^{m} \int_{0}^{t}\left(\gamma(b, n, t-z)-w_{\infty}(b, n, t-z)\right) \varpi(u, m-n, z) d z d t
\end{aligned}
$$

or equivalently that

$$
\sum_{n=1}^{m} \int_{0}^{t} \varsigma(b, m-n, t-z) w_{1}(u, n, z) d z=\sum_{n=1}^{m} \int_{0}^{t} \varsigma(b, m-n, t-z) w_{\infty}(u, n, z)
$$




$$
+\left(\gamma(b, n, t-z)-w_{\infty}(b, n, t-z)\right) \varpi(u, m-n, z) d z .
$$

Now, if $m=1$ then

$$
\int_{0}^{t} \varsigma(b, 0, t-z) w_{1}(u, 1, z) d z=\vartheta(u, 1,1, t) .
$$

In this case

$$
\begin{aligned}
\int_{0}^{t} \delta_{-b / c_{1}}(t-z) e^{\frac{\lambda b}{c_{1}}} w_{1}(u, 1, z) d z & =e^{\frac{\lambda b}{c_{1}}} w_{1}\left(u, 1, t+\frac{b}{c_{1}}\right) \\
& =\frac{\lambda}{c_{2}} e^{-\lambda t} \bar{F}\left(c_{2} t+b+\frac{c_{2}}{c_{1}} u\right)
\end{aligned}
$$

and

$$
w_{1}(u, 1, t)=\frac{\lambda}{c_{2}} e^{-\lambda\left(t+\frac{b}{c_{1}}\right)} \bar{F}\left(c_{2} t+b+\frac{c_{2}}{c_{1}}(u-b)\right) .
$$

Similarly, if $m=2$ then

$$
\int_{0}^{t} \delta_{-b / c_{1}}(t-z) e^{\frac{\lambda b}{c_{1}}} w_{1}(u, 2, z) d z=\sum_{n=1}^{2} \vartheta(u, 2, n, t)-\int_{0}^{t} \varsigma(b, 1, t-z) w_{1}(u, 1, z) d z
$$

and

$$
w_{1}(u, 2, t)=e^{-\frac{\lambda b}{c_{1}}}\left[\sum_{n=1}^{2} \vartheta\left(u, 2, n, t-\frac{b}{c_{1}}\right)-\int_{0}^{t-\frac{b}{c_{1}}} \varsigma\left(b, 1, t-\frac{b}{c_{1}}-z\right) w_{1}(u, 1, z) d z\right] .
$$

Similarly we can prove the assertion for any $m>1$.

Theorem 6.4 For $u>b$ and $m>1$ the joint density of the number of claims until ruin $N_{\tau}$ and the time to ruin $\tau$ is given by

$$
w_{2}(u, m, t)=\left(\frac{\lambda}{c_{2}}\right)^{m} \sum_{k=0}^{m-1} \sum_{n=0}^{m-k-1} \int_{0}^{u-b} \int_{0}^{t} \int_{0}^{c_{2} z} g_{2}(y, k, z) b_{m-k-n-1}(u-b-x, y) \varepsilon(x, n, t-z) d y d z d x
$$

where

$$
\begin{aligned}
\varepsilon(u, 0, t):= & \int_{u+b}^{c_{2} t+u+b} \bar{F}(y) g_{2}(y-u-b, 0, t) d y \\
\varepsilon(u, m, t):= & \sum_{n=1}^{m} \int_{u}^{u+b} \int_{0}^{t} w_{1}(u+b-x, n, t-z) \int_{x}^{c_{2} z+x} f(y) g_{2}(y-x, m-n, z) d y d z d x \\
& +\int_{u+b}^{c_{2} t+u+b} \bar{F}(y) g_{2}(y-u-b, m, z) d y, \quad n \geq 1 .
\end{aligned}
$$

Proof. To obtain an expression for $w_{2}(u, m, t)$ we first consider $h(x)$ defined in (18). Using (39) we can derive:

$$
h(u)=\int_{u}^{u+b} \sum_{m=1}^{\infty} r^{m} \int_{0}^{\infty} e^{-\delta t} w_{1}(u+b-x, m, t) d t
$$




$$
\begin{aligned}
& \times \sum_{n=0}^{\infty} r^{n} \int_{0}^{\infty} e^{-\delta z} \int_{x}^{c_{2} z+x} f(y) g_{2}(y-x, n, z) d y d z d x \\
& +\sum_{n=0}^{\infty} r^{n} \int_{0}^{\infty} e^{-\delta t} \int_{u+b}^{c_{2} t+u+b} \bar{F}(y) g_{2}(y-u-b, n, t) d y d t \\
= & \sum_{n=1}^{\infty} r^{n} \int_{0}^{\infty} e^{-\delta t}\left[\sum_{m=1}^{n} \int_{u}^{u+b} \int_{0}^{t} w_{1}(u+b-x, m, t-z)\right. \\
& \times \int_{x}^{c_{2} z+x} f(y) g_{2}(y-x, n-m, z) d y d z d x \\
& \left.+\int_{u+b}^{c_{2} t+u+b} \bar{F}(y) g_{2}(y-u-b, n, z) d y\right] d t+\int_{0}^{\infty} e^{-\delta t} \int_{u+b}^{c_{2} t+u+b} \bar{F}(y) g_{2}(y-u-b, 0, t) d y d t \\
= & \sum_{n=0}^{\infty} r^{n} \int_{0}^{\infty} e^{-\delta t} \varepsilon(u, n, t) d t .
\end{aligned}
$$

Moreover, substituting (43), (51) and (38) into (17) gives:

$$
\begin{aligned}
\phi_{2}(u)= & \sum_{m=0}^{\infty}\left(\frac{\lambda r}{c_{2}}\right)^{m+1} \int_{0}^{u-b}\left(T_{\rho_{2}} f\right)^{m *}(u-b-x) h(x) d x \\
= & \sum_{m=0}^{\infty}\left(\frac{\lambda r}{c_{2}}\right)^{m+1} \int_{0}^{u-b} \int_{0}^{\infty} e^{-\rho_{2} y} b_{m}(u-b-x, y) d y \sum_{n=0}^{\infty} r^{n} \int_{0}^{\infty} e^{-\delta t} \varepsilon(x, n, t) d t d x \\
= & \sum_{m=0}^{\infty}\left(\frac{\lambda r}{c_{2}}\right)^{m+1} \int_{0}^{u-b} \int_{0}^{\infty} \sum_{k=0}^{\infty} r^{k} \int_{y / c_{2}}^{\infty} e^{-\delta z} g_{2}(y, k, z) d z b_{m}(u-b-x, y) d y \\
& \times \sum_{n=0}^{\infty} r^{n} \int_{0}^{\infty} e^{-\delta t} \varepsilon(x, n, t) d t d x \\
= & \sum_{m=1}^{\infty} r^{m} \int_{0}^{\infty} e^{-\delta t}\left\{\left(\frac{\lambda}{c_{2}}\right)^{m} \sum_{k=0}^{m-1} \sum_{n=0}^{m-k-1} \int_{0}^{u-b} \int_{0}^{t} \int_{0}^{c_{2} z} g_{2}(y, k, z) b_{m-k-n-1}(u-b-x, y)\right. \\
& \times \varepsilon(x, n, t-z) d y d z d x\} d t .
\end{aligned}
$$

Comparing equations (52) and (4) completes the proof.

\section{Acknowledgements}

This research is support by the National Natural Science Foundation of China (Grant No. 11271164) and by the FP7 Grant PIRSES-GA-2012-318984. Zbigniew Palmowski is supported by the National Science Centre under the grant 2015/17/B/ST1/01102 (2016-2019).

\section{References}

[1] A. Badescu, S. Drekic, and D. Landriault. Analysis of a threshold dividend strategy for a MAP risk model. Scandinavian Actuarial Journal, 2007(4):227-247, 2007.

[2] B. De Finetti. Su un'impostazione alternativa della teoria collettiva del rischio. In Transactions of the XVth international congress of Actuaries, pages 433-443, 1957. 
[3] D. C. M. Dickson. The joint distribution of the time to ruin and the number of claims until ruin in the classical risk model. Insurance: Mathematics and Economics, 50(3):334-337, 2012.

[4] D. C. M. Dickson and C. Hipp. On the time to ruin for Erlang (2) risk processes. Insurance: Mathematics and Economics, 29(3):333-344, 2001.

[5] D. C. M. Dickson and G. E. Willmot. The density of the time to ruin in the classical Poisson risk model. Astin Bulletin, 35(1):45-60, 2005.

[6] E Frostig, S. M. Pitts, and K. Politis. The time to ruin and the number of claims until ruin for phase-type claims. Insurance: Mathematics and Economics, 51(1):19-25, 2012.

[7] H. Gao and C. Yin. The perturbed Sparre Andersen model with a threshold dividend strategy. Journal of Computational and Applied Mathematics, 220(1-2):394-408, 2008.

[8] H. U. Gerber and E. S. W. Shiu. The time value of ruin in a Sparre Andersen model. North American Actuarial Journal, 9(2):49-69, 2005.

[9] A. E. Kyprianou and R. L. Loeffen. Refracted Lévy processes. Annales De L' Institut Henri Poincaré Probabilités Et Statistiques, 46(1):24-44, 2010.

[10] J. L. Lagrange. Nouvelle méthode pour résoudre les équations littérales par le moyen des séries. Chez Haude et Spener, Libraires de la Cour \& de l'Acadmie royale, 1770.

[11] D. Landriault, T. Shi, and G. E. Willmot. Joint densities involving the time to ruin in the Sparre Andersen risk model under exponential assumptions. Insurance: Mathematics and Economics, 49(3):371-379, 2011.

[12] S. Li and J. Garrido. On a class of renewal risk models with a constant dividend barrier. Insurance: Mathematics and Economics, 35(3):691-701, 2004.

[13] S. Li and J. Garrido. On ruin for the Erlang (n) risk process. Insurance: Mathematics and Economics, 34(3):391-408, 2004.

[14] S. Li and Y. Lu. The distribution of total dividend payments in a Sparre Andersen model. Statistics and Probability Letters, 79(9):1246-1251, 2009.

[15] S. Li and Y. Lu. On the time and the number of claims when the surplus drops below a certain level. Scandinavian Actuarial Journal, 5: 420-445, 2016.

[16] Y. Lu and S. Li. The Markovian regime-switching risk model with a threshold dividend strategy. Insurance: Mathematics and Economics, 44(2):296-303, 2009.

[17] X. S. Lin and K. P. Pavlova. The compound Poisson risk model with a threshold dividend strategy. Insurance: Mathematics and Economics, 38(1):57-80, 2006. 
[18] X. S. Lin, G. E. Willmot, and S. Drekic. The classical risk model with a constant dividend barrier: analysis of the Gerber-Shiu discounted penalty function. Insurance: Mathematics and Economics, 33(3):551-566, 2003.

[19] G. E. Willmot and D. C. M. Dickson. The Gerber-Shiu discounted penalty function in the stationary renewal risk model. Insurance: Mathematics and Economics, 32(3):403-411, 2003.

[20] C. Zhao and C. Zhang. Joint density of the number of claims until ruin and the time to ruin in the delayed renewal risk model with Erlang (n) claims. Journal of Computational and Applied Mathematics, 244:102-114, 2013.

[21] X. Zhou. On a classical risk model with a constant dividend barrier. North American Actuarial Journal, 9(4):95-108, 2005.

[22] J. Zhu and H. Yang. Ruin theory for a Markov regime-switching model under a threshold dividend strategy. Insurance: Mathematics and Economics, 42(1):311-318, 2008. 